\title{
Characterization of AAm/4-Styrenesulfonic Acid Sodium Salt/PEG Hydrogels Crosslinked by PEGDA as Highly Swollen Adsorbents for the Effective Removal of Janus Green B from Aqueous Solutions
}

\author{
Ömer Barış ÜZüm, Erdener Karadağ* (1) \\ Department of Chemistry, Fen-Edebiyat Faculty, Aydın Adnan Menderes University, Aydın, Turkey \\ Email: *ekaradag@adu.edu.tr
}

How to cite this paper: ÜZüm, Ö.B. and Karadağ, E. (2021) Characterization of AAm/ 4-Styrenesulfonic Acid Sodium Salt/PEG Hydrogels Crosslinked by PEGDA as Highly Swollen Adsorbents for the Effective Removal of Janus Green B from Aqueous Solutions. Journal of Encapsulation and Adsorption Sciences, 11, 45-68.

https://doi.org/10.4236/jeas.2021.112003

Received: April 14, 2021

Accepted: June 5, 2021

Published: June 8, 2021

Copyright $\odot 2021$ by author(s) and Scientific Research Publishing Inc. This work is licensed under the Creative Commons Attribution International License (CC BY 4.0).

http://creativecommons.org/licenses/by/4.0/

\begin{abstract}
In this study, it has been investigated that the incorporation of poly(ethylene glycol) and 4-styrenesulfonic acid sodium salt into acrylamide hydrogel during free radical solution polymerization synthesis for novel highly swollen adsorbents and for the effective removal of some potential pollutants from aqueous solutions such as cationic dyes. Poly(ethylene glycol)diacrylate was used as a multifunctional crosslinker. The main purpose of this study was to combine both monomers and a polymer in a new polymeric system. Dye sorption properties of the polymeric hydrogels were investigated by using cationic dye such as Janus Green B. Swelling and sorption studies were carried out at $25^{\circ} \mathrm{C}$. For structural characterization, FT-IR analysis and SEM studies were applied. To determine the sorption behaviors of Janus Green B, some sorption parameters such as dye removal capacity, adsorption percentage, and partition coefficient of the polymeric hydrogels were investigated.
\end{abstract}

\section{Keywords}

Hydrogel, Swelling, Composite, Acrylamide, Dye Sorption

\section{Introduction}

Hydrogels are cross-linked, three-dimensional networked polymers that can swell without dissolving in water. Hydrogels with a water content of $100 \%$ more than their mass are called "super absorbents". In order for a polymer to be a hydrogel, hydrogel groups that can form hydrogen bonds such as hydroxyl, car- 
boxyl, carbonyl, amine, and amide must be present in its main chain or side branches. They are three-dimensional reticulated polymers that do not dissolve when contacted with the aqueous environment, show swelling property by holding large amounts of water, and contain many hydrophilic groups. They are also called "hungry net structures" due to their high water-holding properties. Having unique properties such as the ease of preparation in different geometric shapes, high water content, soft and rubbery structure, compatibility with biological fluids, and maximum compatibility with the surrounding tissues allow hydrogels to be used as biomaterials [1]-[7].

In recent years, the importance of hydrogels has increased considerably and they have a wide range of uses [1] [2] [3] [4]. Due to these properties, hydrogels, which are versatile materials, have a wide range of applications in biomedicine and pharmacy studies. Hydrogels are used in fields such as biotechnology, bioengineering, biomedical sciences, pharmacy due to their ability to hold water very well and swell in water/aqueous environments [5] [6] [7].

In order to develop mechanically resistant polymers, "Interpenetrating Polymer Network, IPN", which are three-dimensional network structures, in which one or all of the polymers that form it are cross-linked, can be used. Since there is no chemical bond between the polymers that make up the IPN, both components preserve their own properties and can create a structure with the required conditions. If one of the polymers that make up IPN is the straight chain, such structures are called semi-IPN. Semi-IPN hydrogels also contain one of the structures, while the other does not. In the production of IPNs, it can be used in natural polymers such as carboxymethyl cellulose, sodium alginate, xantham gum, gelatin, chitin, chitosan, as well as artificial polymers such as poly(vinyl alcohol), poly(ethylene glycol), polyacrylamide, poly(N-isopropyl alcohol). By combining these polymers, IPN structures with different properties can be formed. IPNs are three-dimensional mesh structures in which one or all of the polymers that compose it are cross-linked. Each network structure that makes up the IPN can be created at the same time or later. IPNs are structures that can exhibit swelling or shrinkage depending on $\mathrm{pH}$ and ionic strength. These network structures can carry both acidic and basic groups attached to their chains. In aqueous environments with appropriate $\mathrm{pH}$ and ionic strength, these groups form a fixed electrical charge (negative or positive) in the network structure by ionizing. As a result of the effect of these electrostatic forces, the solvent entry into the mesh may increase and the structure may swell, as well as the structure may show shrinkage behavior by decreasing the solvent input [6] [8] [9].

Crosslinked or mesh polymers are very suitable for absorption due to their high water-holding ability, water purification, heavy metal/dyestuff removal, chromatographic applications, ion exchange applications, solvent extraction processes. Crosslinked polymers or copolymers can be used for removal of water from petroleum and oil-containing industrial waste mixtures, for inspection of water-containing hospital wastes, paint, and heavy metal-containing industrial wastewater, for prevention of corrosion in telecommunications. Hydrogels can 
be used to remove heavy metal ions and dyestuffs from aqueous environments by the surface absorption method. The absorption properties of the networked polymers to be used for this purpose are determined by adsorption studies carried out at constant temperature. With the help of adsorption isotherms, parameters such as adsorption capacity, percent adsorption, and dispersion coefficient can be calculated. In many previous studies, several kinds of water absorbent composites based on some clay or synthetic polymers were prepared, and these superabsorbent composites showed high water absorbency and water retention, good salt-resistance, and low production costs in comparison with pure organic superabsorbent polymers under the same preparation condition [10] [11].

Polyacrylamide based hydrogels have received considerable attention because of their use in many applications (as specific sorbent, etc.) [12]-[17]. In our previous studies, copolymeric hydrogels of acrylamide with some acidic monomers were prepared by free radical solution polymerization and used in the separation and adsorption of some dye molecules [3] [6] [12] [13] [15]. It was reported that a lot of 4-styrenesulfonic acid sodium salt, (SSS) based studies by free radical polymerization [5] [18]. In these studies, it was described that strongly charged property of SSS at some polyelectrolyte copolymers. Poly (ethylene glycol), $(\mathrm{PEG})$ is of great interest in numerous biomedical applications for several purposes. PEG based hydrogels have good biocompatibility. PEG based hydrogel systems have been used in many biotechnological applications [19] [20] [21].

In this study, it was of interest to increase/decrease the water and dye sorption capacity of AAm hydrogels with highly hydrophilic functional groups containing chemical reagents such as SSS with PEG via free radical solution polymerization method. AAm is a highly hydrophilic monomer, SSS is anionic and strongly charged monomer and PEG is linear polymer. The main purpose of this study was to combine both monomers and a polymer in a new polymeric system. In this respect, a series of AAm based copolymeric hydrogels were synthesized by changing the content of SSS and PEG. Then, some swelling, and some diffusional properties of AAm/SSS hydrogels and AAm/SSS/PEG semi IPNs were studied in water by swelling studies for swelling characterization. Water uptake and dye sorption properties of AAm/SSS hydrogels and AAm/SSS/PEG semi IPNs were investigated as a function of chemical composition of the hydrogels.

\section{Materials and Methods}

\subsection{Raw Materials}

Acrylamide (AAm), the initiator, ammonium persulphate (APS), supplied by Merck, (Darmstad, Germany), the activator $N, N, N$, $N$ '-tetramethylethylenediamine (TEMED) were supplied by Merck (Schuchardt, Germany). Anionic co-monomer, 4-styrenesulfonic acid sodium salt (SSS) and poly(ethylene glycol) (PEG, $M_{w}=$ 4000), were supplied by Fluka Chemical Co., (Steinheim, Germany). Poly(ethylene glycol)diacrylate (PEGDA, $M_{n}=700$ ) as crosslinker were supplied from Aldrich, (Steinheim, Germany). 
Cationic dye, Janus Green B, (JGB) used in sorption studies, were purchased from Fluka, Steinheim, Germany. Some properties of JGB were presented in Table 1. All chemicals were used as received.

\subsection{Preparation of AAm/SSS Hydrogels and AAm/SSS/PEG Semi IPNs}

To prepare AAm/SSS hydrogel systems, AAm weighing $1.0 \mathrm{~g}(14.07 \mathrm{mmol})$ was dissolved in $1.0 \mathrm{~mL}$ water. Then $0 \mathrm{mg}, 10 \mathrm{mg} / 0.0485 \mathrm{mmol}, 20 \mathrm{mg} / 0.0970 \mathrm{mmol}$, $30 \mathrm{mg} / 0.145 \mathrm{mmol}, 40 \mathrm{mg} / 0.194 \mathrm{mmol}, 50 \mathrm{mg} / 0.242 \mathrm{mmol}, 60 \mathrm{mg} / 0.291 \mathrm{mmol}, 70$ $\mathrm{mg} / 0.339 \mathrm{mmol}, 80 \mathrm{mg} / 0.388 \mathrm{mmol}$ of SSS were added to each AAm solutions, respectively. After these additions, for the synthesis, $0.040 \mathrm{~mL} / 0.0640 \mathrm{mmol}$ of PEGDA and $0.2 \mathrm{~mL} / 0.0438 \mathrm{mmol}$ aqueous solutions of APS (5.0 g APS/0.022 $\mathrm{mol} / 100 \mathrm{~mL}$ water) and $0.25 \mathrm{~mL} / 0.0167 \mathrm{mmol} 1 \%$ concentration of TEMED were added these aqueous solutions. The solutions were placed in PVC straws of 3 $\mathrm{mm}$ diameter. After gelation, fresh hydrogels obtained in long cylindrical shapes were cut into pieces of $3-4 \mathrm{~mm}$ in length. They were washed 4 days in distilled water to remove unreacted materials, blotted dry with filter paper, dried in air and vacuum, and stored for swelling and sorption studies.

To prepare highly swollen AAm/SSS/PEG semi IPNs, same method was used as mentioned above with addition of $0.25,0.50,0.75$ and $1.00 \mathrm{~g}$ PEG to aqueous monomer solution per $1.0 \mathrm{~g}$ of AAm.

For swelling studies, AAm/SSS hydrogels and AAm/SSS/PEG semi IPNs were accurately weighed and transferred into water. Water uptake with respect to time was obtained by periodically removing the samples from water; quickly blot drying, and reweighing. The measurements were conducted at $25^{\circ} \mathrm{C} \pm 0.1^{\circ} \mathrm{C}$ in a water bath.

\subsection{FT-IR Analysis of AAm/SSS Hydrogels and AAm/SSS/PEG Semi IPNs}

For structural characterization, FT-IR analysis was made. Spectra were taken on $\mathrm{KBr}$ discs by using VARIAN FTS 800 FT-IR spectrophotometer.

\subsection{Surface Morphology of AAm/SSS Hydrogels and AAm/SSS/PEG Semi IPNs}

The surface morphology of AAm/SSS hydrogels and AAm/SSS/PEG semi IPNs

Table 1. Some properties of Janus Green B.

Name
(JGB)
(Union green B)


was visualized by a scanning electronic microscope PHILLIPS XL-30S FEG, FEI QUANTA 250 FEG. The surface of the AAm/SSS hydrogels and AAm/SSS/PEG semi IPNs was coated with gold under vacuum using EMITECH K550X.

\subsection{Sorption Studies of AAm/SSS Hydrogels and AAm/SSS/PEG Semi IPNs}

Batch sorption studies were applied in all sorption experiments. Cationic dye, Janus Green B, (JGB) used in sorption studies and some properties of JGB were given in Table 1. Solutions of JGB concentration range $1.25 \times 10^{-3} \mathrm{M}-2.00 \times$ $10^{-3} \mathrm{M}$ in distilled water were prepared. AAm/SSS hydrogels and AAm/SSS/PEG semi IPNs containing $60 \mathrm{mg}$ SSS was used in a known volume of dye solution until equilibrium was reached. For SSS effect on the dye sorption, $2.00 \times 10^{-3} \mathrm{M}$ aqueous solution of JGB was used. After sorption, dye solution was separated by decantation from the hydrogels. Spectrophotometric method was applied to dye solutions. Spectrophotometric measurements were carried out using a SHIMADZU UV 1601 model UV-VIS spectrophotometer at ambient temperature. The absorbance of these solutions was read at $660 \mathrm{~nm}$ for JGB [22]. Distilled water was chosen as the reference. The equilibrium concentrations of the cationic dye solutions were determined by means of precalibrated scales.

\section{Results and Discussion}

\subsection{Preparation}

AAm/SSS hydrogels and AAm/SSS/PEG semi IPNs were prepared by free radical solution polymerization. Dried AAm/SSS hydrogels and AAm/SSS/PEG semi IPNs are glassy and very hard, but swollen gels are soft. SSS received attention in recent years due to its strongly ionizable charged group. The synthesis of AAm/ SSS hydrogels and AAm/SSS/PEG semi IPNs via radical chain polymerization is a well-established procedure. Representative chemical structures of monomers and possible AAm/SSS copolymer have been presented at Figure 1.

\subsection{FT-IR Analysis}

To understand binding and crosslinking of AAm/SSS hydrogels and AAm/SSS/ PEG semi IPNs during polymerization, FT-IR spectra of the hydrogel systems were evaluated and are presented in Figure 2 and Figure 3. In the FT-IR spectra of the hydrogels, the bands at about 1700 and $3100-3500 \mathrm{~cm}^{-1}$ are important. The bands at $1600-1700 \mathrm{~cm}^{-1}$ could be attributed to a shift in stretching vibration associated with hydrogen that is bonded directly to an overtone of the strong carbonyl absorption. The peak at $1650-1660 \mathrm{~cm}^{-1}$ is the carbonyl group and related to amide groups and at $1500-1600 \mathrm{~cm}^{-1}$ is the $\mathrm{N}-\mathrm{H}$ bonding vibration. The much broader absorption peaks in the regions of $3100 \mathrm{~cm}^{-1}$ and 3500 $\mathrm{cm}^{-1}$ are N-H bands and related to "polymeric" bands. In Figure 3, there is a group of absorption peaks between $3400-3600 \mathrm{~cm}^{-1}$, which is due to stretching bands of the $\mathrm{OH}$ groups, and the band at $1640 \mathrm{~cm}^{-1}$ also corresponds to the $\mathrm{OH}$ 


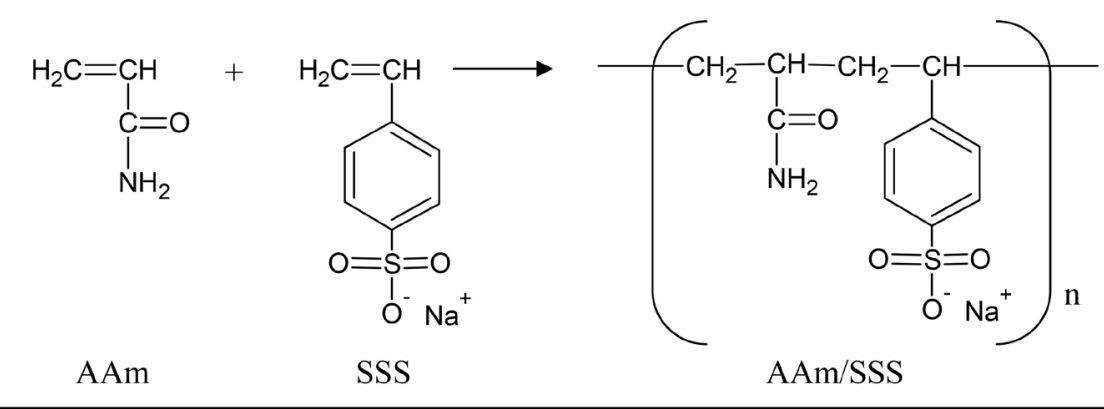

Figure 1. Representative chemical structures of monomers and possible AAm/SSS copolymer.

\section{AAm/SSS}

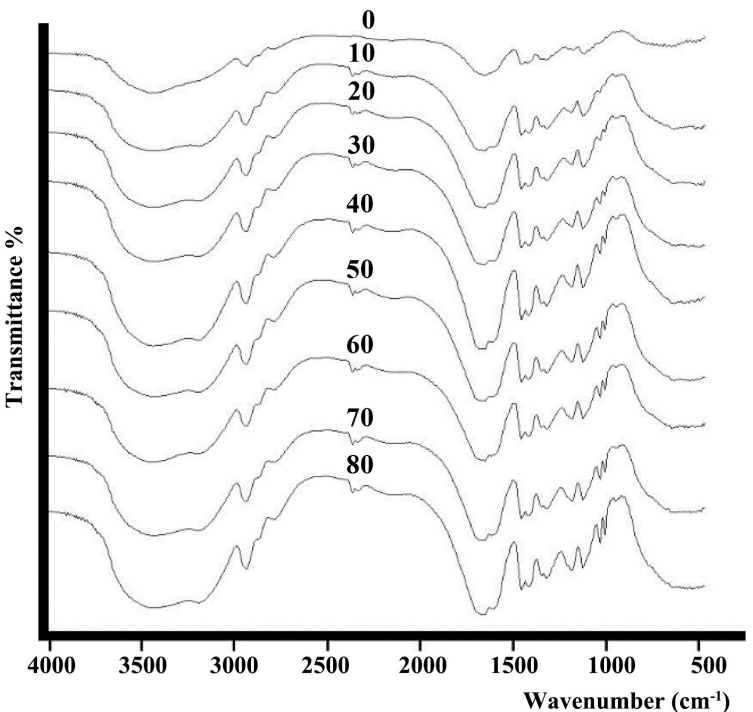

AAm/SSS/PEG

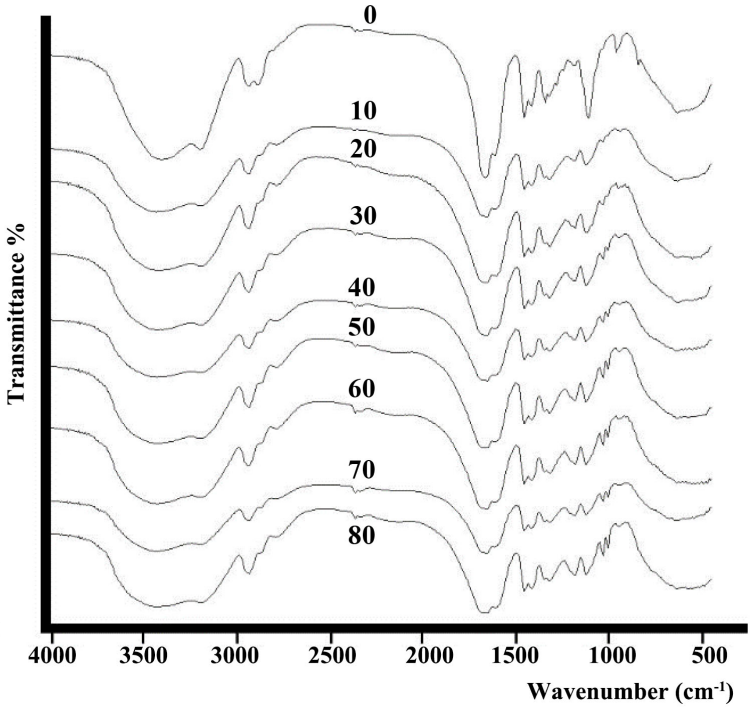

Figure 2. FT-IR spectra of AAm/SSS hydrogels and AAm/SSS/PEG semi IPNs including of various contents as mg SSS.

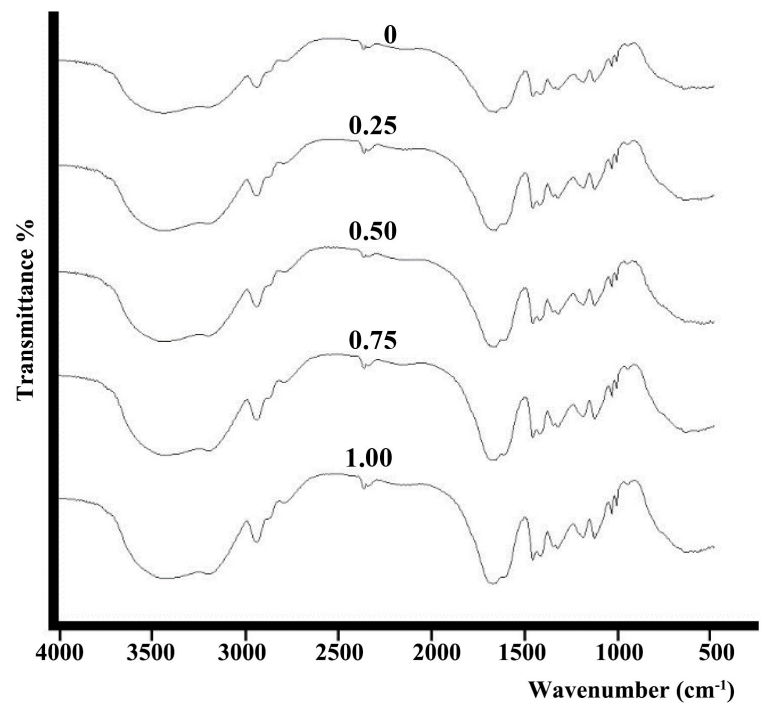

Figure 3. FT-IR spectra of AAm/SSS/PEG semi IPNs including of $60 \mathrm{mg}$ of SSS and various amount of PEG. 
groups. The broad peak 3500 is characteristic peak of primary amine. On the other hand, it is thought that the peaks at $1200 \mathrm{~cm}^{-1}$ are C-N bands, and the peaks at $2850 \mathrm{~cm}^{-1}$ and $1400 \mathrm{~cm}^{-1}$ show $\mathrm{CH}_{2}$ groups on the polymeric chain. The characteristic absorption peak of SSS units is shown at $1040 \mathrm{~cm}^{-1}$ due to $\mathrm{S}=\mathrm{O}$ group [18] [23].

The characteristic absorption peak of PEG units is shown at $1100-1200 \mathrm{~cm}^{-1}$ due to aliphatic ether bonds [23]. The peaks observed in the FT-IR spectra confirm the presence of AAm, SSS and PEG.

\subsection{Equilibrium Swelling Studies}

A fundamental relationship exists between the swelling of a polymer in a solvent and the nature of the polymer and the solvent. The percentage swelling $(S \%)$ of the hydrogels in distilled water was calculated from the following relation,

$$
S \%=\frac{m_{t}-m_{o}}{m_{o}} \times 100
$$

where $m_{t}$ is the mass of the swollen gel at time $t$ and $m_{o}$ is the mass of the dry gel at time 0 .

The water intake of initially dry hydrogels was followed for a period of time, gravimetrically. Swelling isotherms of the hydrogels were constructed and representative swelling curve is shown in Figure 4 and Figure 5.

Figure 4 and Figure 5 show that swelling increase with time up to certain level, and then levels off. This value of swelling may be called the "equilibrium percentage swelling" ( $\left.S_{e q} \%\right)$. The values of $S_{e q} \%$ of AAm/SSS hydrogels and AAm/ SSS/PEG semi IPNs are used for the calculation of network characterization parameters. The values of $S_{e q} \%$ of AAm/SSS hydrogels and AAm/SSS/PEG semi IPNs are given Table 2. Table 2 shows that $S_{e q} \%$ of AAm hydrogels is $490 \%$, but $S_{e q} \%$ of AAm/SSS hydrogels are $530 \%-850 \%$ with the incorporation of SSS groups into AAm hydrogels, and $S_{e q} \%$ of AAm/SSS/PEG semi IPNs are $435 \%$ $790 \%$ with the incorporation of PEG groups into AAm/SSS hydrogels, while $S_{e q} \%$ of AAm/PEG semi IPNs is $420 \%$. In Table $2, S_{e q} \%$ of the hydrogels increased with the SSS content in the copolymers. $S_{e q} \%$ of AAm/SSS hydrogels is

Table 2. Values of the equilibrium percentage swelling $\left(S_{e q} \%\right)$ and equilibrium water contents (EWC) of AAm/SSS hydrogels and AAm/SSS/PEG (containing $0.5 \mathrm{~g}$ PEG) semi IPNs.

\begin{tabular}{|c|c|c|c|c|c|c|c|c|c|}
\hline $\mathrm{SSS} / \mathrm{mg}$ & 0 & 10 & 20 & 30 & 40 & 50 & 60 & 70 & 80 \\
\hline & \multicolumn{9}{|c|}{ Equilibrium percentage swelling $\left(S_{e q} \%\right)$} \\
\hline & 490 & 530 & 590 & 630 & 670 & 710 & 730 & 810 & 850 \\
\hline \multirow[t]{3}{*}{ PEG } & 420 & 435 & 450 & 510 & 620 & 630 & 690 & 740 & 790 \\
\hline & \multicolumn{9}{|c|}{ Equilibrium water content (EWC) } \\
\hline & 0.8312 & 0.8417 & 0.8550 & 0.8623 & 0.8692 & 0.8770 & 0.8800 & 0.8903 & 0.8946 \\
\hline PEG & 0.8063 & 0.8131 & 0.8167 & 0.8360 & 0.8610 & 0.8632 & 0.8739 & 0.8807 & 0.8870 \\
\hline
\end{tabular}




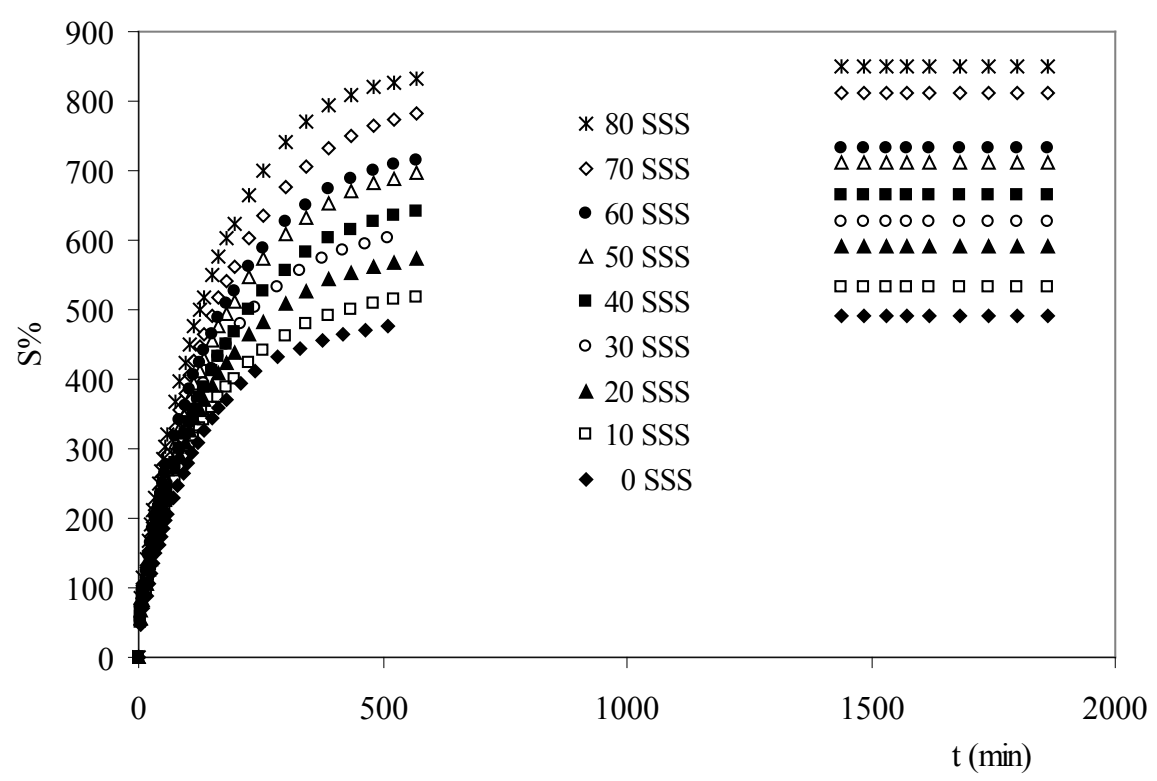

Figure 4. Swelling isotherms of AAm/SSS hydrogels.

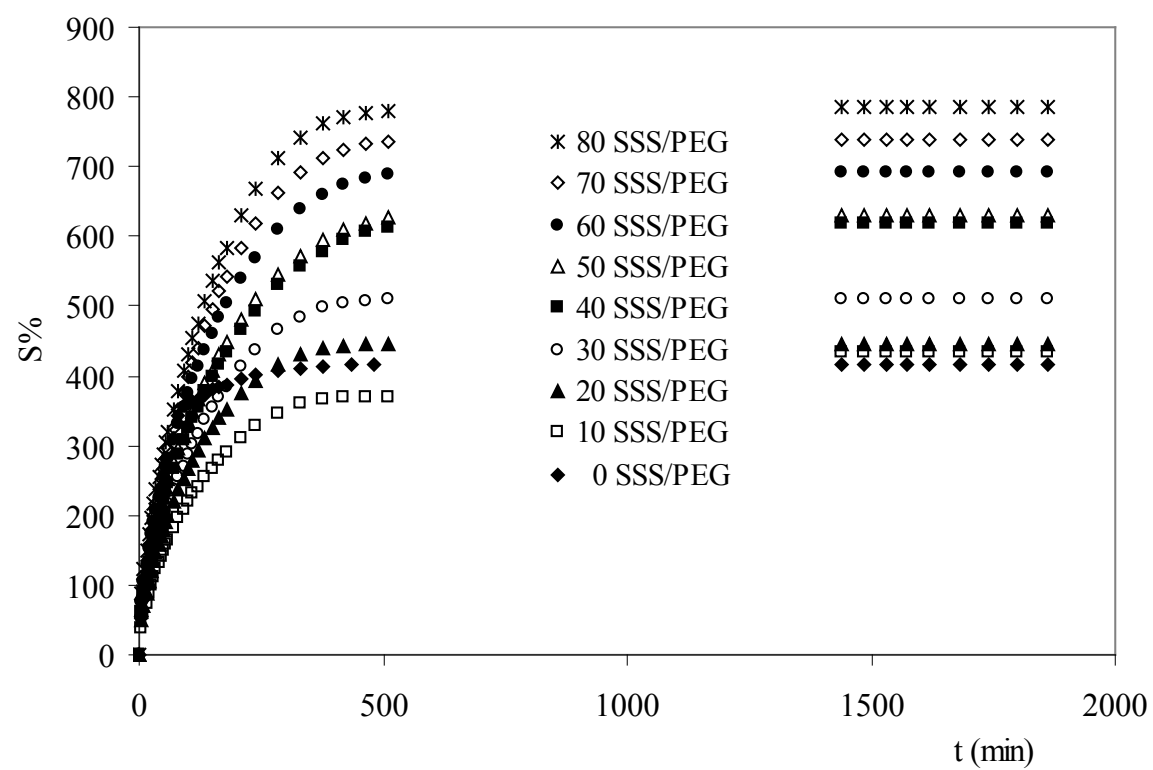

Figure 5. Swelling isotherms of AAm/SSS/PEG semi IPNs.

higher than $S_{e q} \%$ of AAm hydrogels. Table 2 also shows that $S_{e q} \%$ values of AAm/SSS/PEG semi IPNs lower than $S_{e q} \%$ values of AAm/SSS hydrogels. One of the reasons of these results is decreasing of hydrophilic character at crosslinked polymeric systems. Additionally, the PEG chains are located in the free space of crosslinked polymer networks; therefore, water diffusion is prevented by the PEG chains.

It is well known that the swelling of a hydrogel is induced by electrostatic repulsion of the ionic charges of its network. The ionic charge content is important. SSS contains many ionic groups $\left(-\mathrm{SO}_{3} \mathrm{Na}\right.$ ) (Figure 1). The swelling increase is due to an increase in the anionic units. The salt group is almost completely io- 
nized, and a large number of hydrophilic groups occur. The hydrophilic group numbers of AAm/SSS hydrogels and AAm/SSS/PEG semi IPNs are higher than those of AAm, and so the swelling values of AAm/SSS hydrogels and AAm/SSS/ PEG semi IPNs are greater than that of AAm swelling values. Upon swelling the hydrogels were strong enough to retain their shape. There have been presented the photographs of AAm/SSS hydrogels and AAm/SSS/PEG semi IPNs at Figure 6 and Figure 7, as dry state and as swollen state. The capacity of swelling of the hydrogel systems can be seen from their photographs.

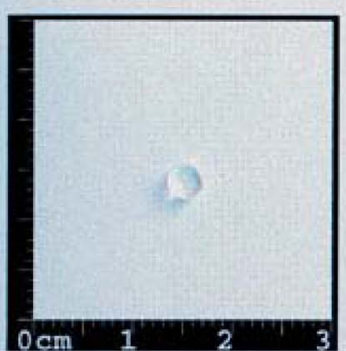

0 SSS

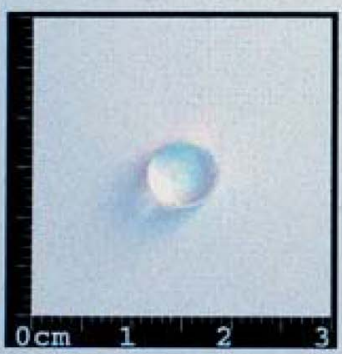

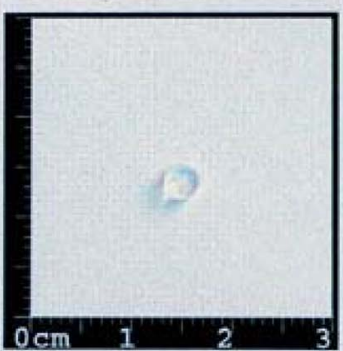

$60 \mathrm{SSS}$

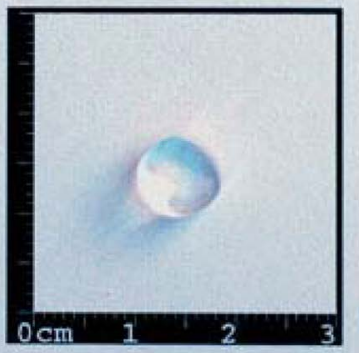

Figure 6. The photographs of AAm/SSS hydrogel (as dry or swollen state).

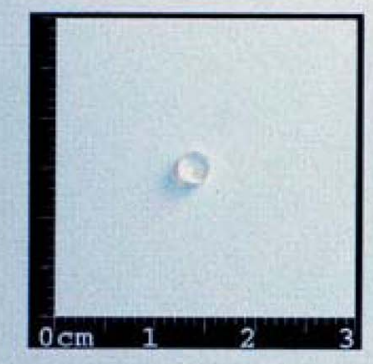

0 SSS/PEG

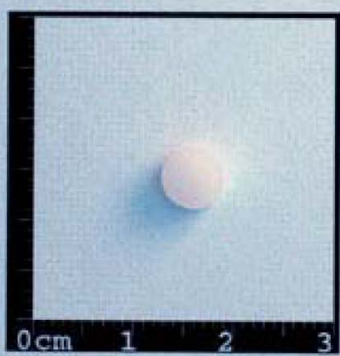

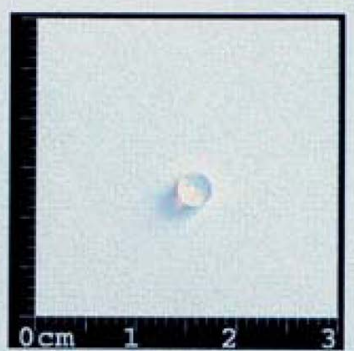

$60 \mathrm{SSS} / \mathrm{PEG}$

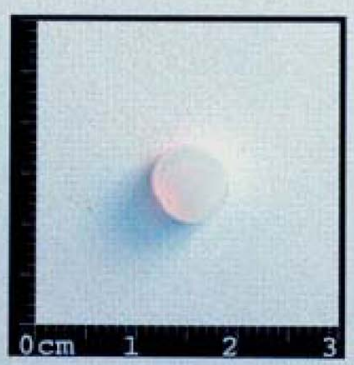

Figure 7. The photographs of AAm/SSS/PEG semi IPNs (as dry or swollen state). 


\subsection{Equilibrium Water Content}

The water absorbed by AAm/SSS hydrogels and AAm/SSS/PEG semi IPNs is quantitatively represented by the equilibrium water content (EWC), by using below equation [24] [25].

$$
\mathrm{EWC}=\frac{m_{s}-m_{o}}{m_{s}}
$$

Here, $m_{s}$ is the mass of the swollen gel at time $t$ (equilibrium), and $m_{o}$ is the mass of the dry gel at time 0 . The EWC values of all AAm/SSS hydrogels and AAm/ SSS/PEG semi IPNs were calculated. The values of EWC of the hydrogels are tabulated in Table 2. Generally, it is seen that an increasing of the values of EWC, if SSS have been added to composite hydrogel systems. Here, the main effect is the hydrophilic character of SSS groups.

\subsection{Swelling Kinetics}

In order to examine the controlling mechanism of the swelling processes, several kinetic models are used to test experimental data. The large number and array of different chemical groups on the polymer chains (e.g., amine, amide, carbonyl, carboxyl or hydroxyl) imply that there are many types of polymer-solvent interactions. It is probable that any kinetics is likely to be global. From a system design viewpoint, a lumped analysis of swelling rates is thus sufficient to the practical operation.

A simple kinetic analysis is a second-order equation in the form of

$$
\frac{\mathrm{d} S}{\mathrm{~d} t}=k_{S}(S-S t)^{2}
$$

where $k_{s}$ is the rate constant of swelling and $S$ denotes the degree of swelling at equilibrium [26] [27]. After definite integration by applying the initial conditions $S=0$ at $t=0$ and $S_{t}=S$ at $t=t$, Equation (3) becomes

$$
\frac{t}{S}=A+B t
$$

Here $A$ is reciprocal of initial swelling rate $r_{o}$ or $1 / k_{s} S^{2}$ and $B$ is inverse of the degree of swelling at equilibrium.

To test the kinetics model, $t / S$ vs. $t$ graphs are plotted and representative graph is illustrated in Figure 8 and Figure 9. The calculated kinetic parameters are tabulated in Table 3.

As can be seen from Table 3, kinetics model is agreement with swelling experiments, since, as depicted in Table 2, Percentage swelling, $S \%\left(S_{e q} \%\right)$ is changed with SSS and PEG content. Again, the initial swelling rate is changed with SSS content. This may be plausible since the ionic content of the network is enhanced with the extent of SSS groups in structure. It may be important to note that extent of SSS determines the swelling rate by increasing hydrophilicity.

\subsection{Diffusion}

When a glassy hydrogel is brought into contact with water, water diffuses into 


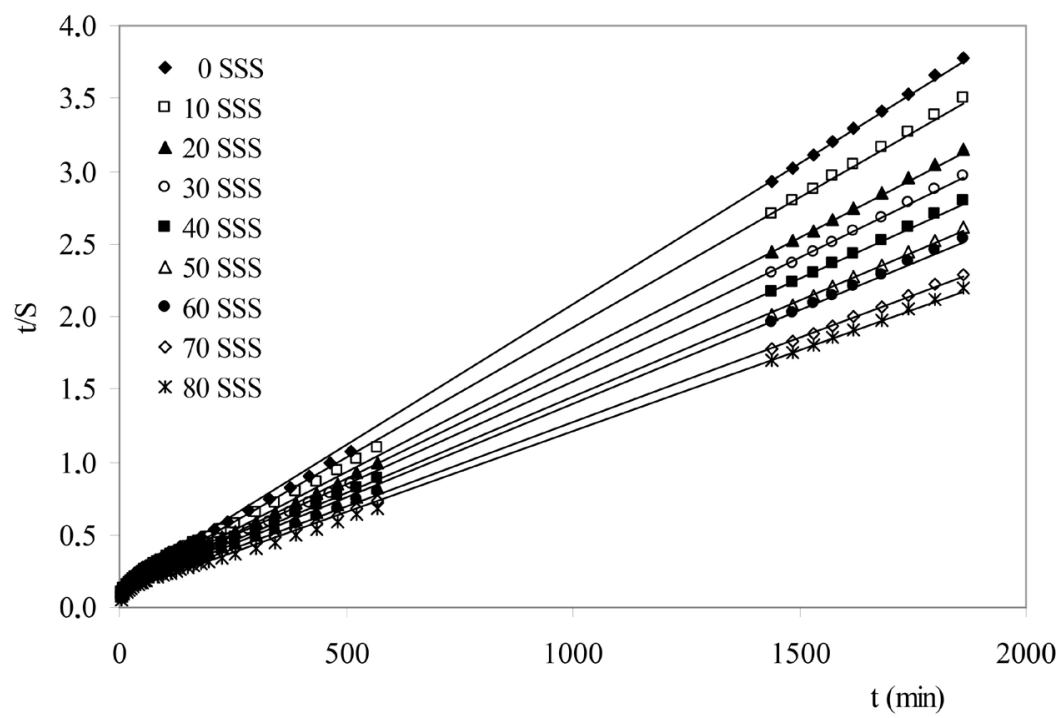

Figure 8. Swelling rate curves of AAm/SSS hydrogels.

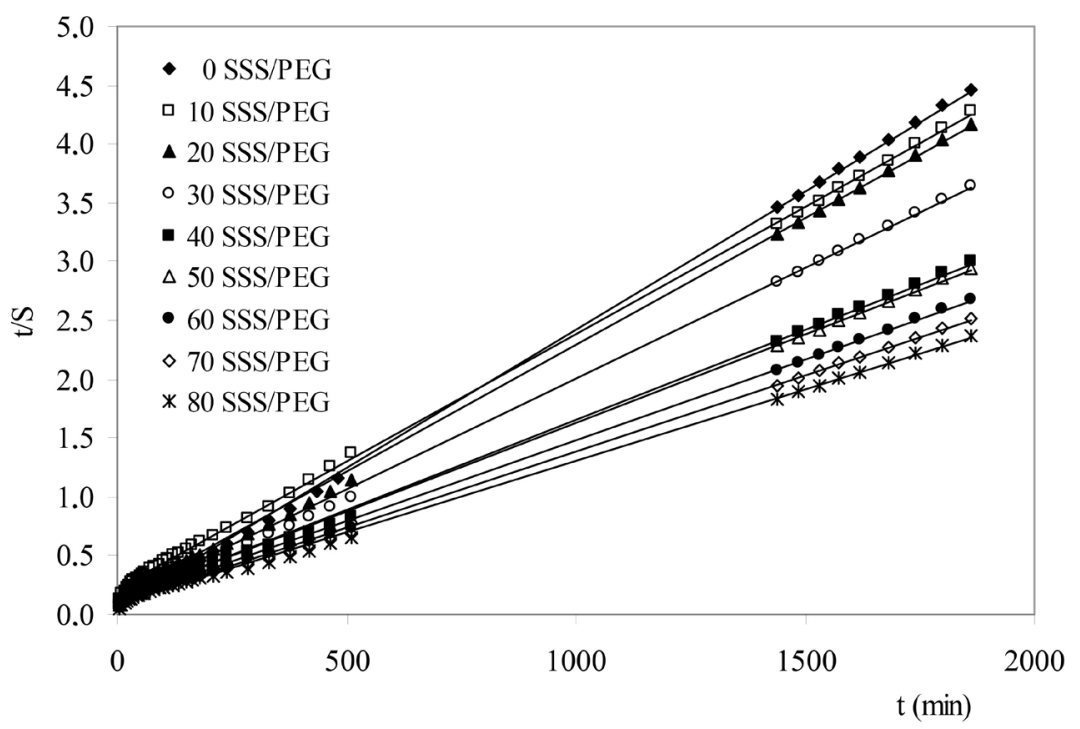

Figure 9. Swelling rate curves of AAm/SSS/PEG semi IPNs.

Table 3. Swelling kinetics parameters of AAm/SSS hydrogels and AAm/SSS/PEG (containing $0.5 \mathrm{~g}$ PEG) semi IPNs.

\begin{tabular}{|c|c|c|c|c|c|c|c|c|c|}
\hline $\mathrm{SSS} / \mathrm{mg}$ & 0 & 10 & 20 & 30 & 40 & 50 & 60 & 70 & 80 \\
\hline & \multicolumn{9}{|c|}{ Initial swelling rate $\left(r_{o}\right) ;(\mathrm{d} S / \mathrm{d} t)_{o} g_{\text {water }} / g_{\text {gel }} \min$} \\
\hline & 7.00 & 7.42 & 7.78 & 8.07 & 7.42 & 8.49 & 8.75 & 8.73 & 10.44 \\
\hline \multirow[t]{3}{*}{ PEG } & 14.53 & 4.65 & 7.66 & 7.78 & 7.93 & 8.26 & 9.59 & 10.25 & 11.37 \\
\hline & \multicolumn{9}{|c|}{ Swelling rate constant $\left(k_{s} \times 10^{5}\right) \cdot g_{\text {gel }} / g_{\text {water }} \min$} \\
\hline & 2.63 & 2.38 & 2.02 & 1.86 & 1.49 & 1.49 & 1.46 & 1.17 & 1.30 \\
\hline \multirow[t]{3}{*}{ PEG } & 8.06 & 2.19 & 3.57 & 2.74 & 1.86 & 1.88 & 1.82 & 1.71 & 1.68 \\
\hline & \multicolumn{9}{|c|}{ Theoretical equilibrium percentage swelling $\left(S_{\max } \%\right) g_{\text {water }} / g_{\text {gel }}$} \\
\hline & 516 & 558 & 620 & 658 & 705 & 754 & 775 & 864 & 897 \\
\hline PEG & 424 & 461 & 464 & 533 & 652 & 664 & 727 & 775 & 822 \\
\hline
\end{tabular}


the hydrogel and the network expand resulting in swelling of the hydrogel. Diffusion involves migration of water into pre-existing or dynamically formed spaces between hydrogel chains. Swelling of the hydrogel involves larger segmental motion resulting, ultimately, in increased separation between hydrogel chains.

Analysis of the mechanisms of water diffusion into swellable polymeric systems has received considerable attention in recent years, because of important applications of swellable polymers in biomedical, pharmaceutical, environmental, and agricultural engineering.

The following equation is used to determine the nature of diffusion of water into hydrogels [27] [28] [29].

$$
F=\frac{M_{t}}{M_{s}}=k t^{n}
$$

Here, $F$ is the fractional uptake at time $t$. Here, $M_{t}$ and $M_{s}$ are the mass uptake of the solvent at time $t$ and the equilibrium, respectively. Equation (5) is valid for the first $60 \%$ of the fractional uptake. Fickian diffusion and Case II transport are defined by $n$ values of 0.5 and 1.0, respectively. Anomalous transport behavior (non-Fickian diffusion) is intermediate between Fickian and Case II. That is reflected by $n$ between 0.5 and 1.0 [27] [28] [29]. The values of $(n)$ and $(k)$ were calculated from the slope and the intercept of the plot of $\ln F$ against $\ln t$, respectively.

For AAm/SSS hydrogel and AAm/SSS/PEG semi IPNs, $\ln F$ vs. $\ln t$ graphs are plotted and representative results are shown in Figure 10 and Figure 11. Diffusional exponents, $(n)$ and diffusion constant, $(k)$ are calculated and listed in Table 4 .

Table 4 shows that the number determining the type of diffusion $(n)$ is over

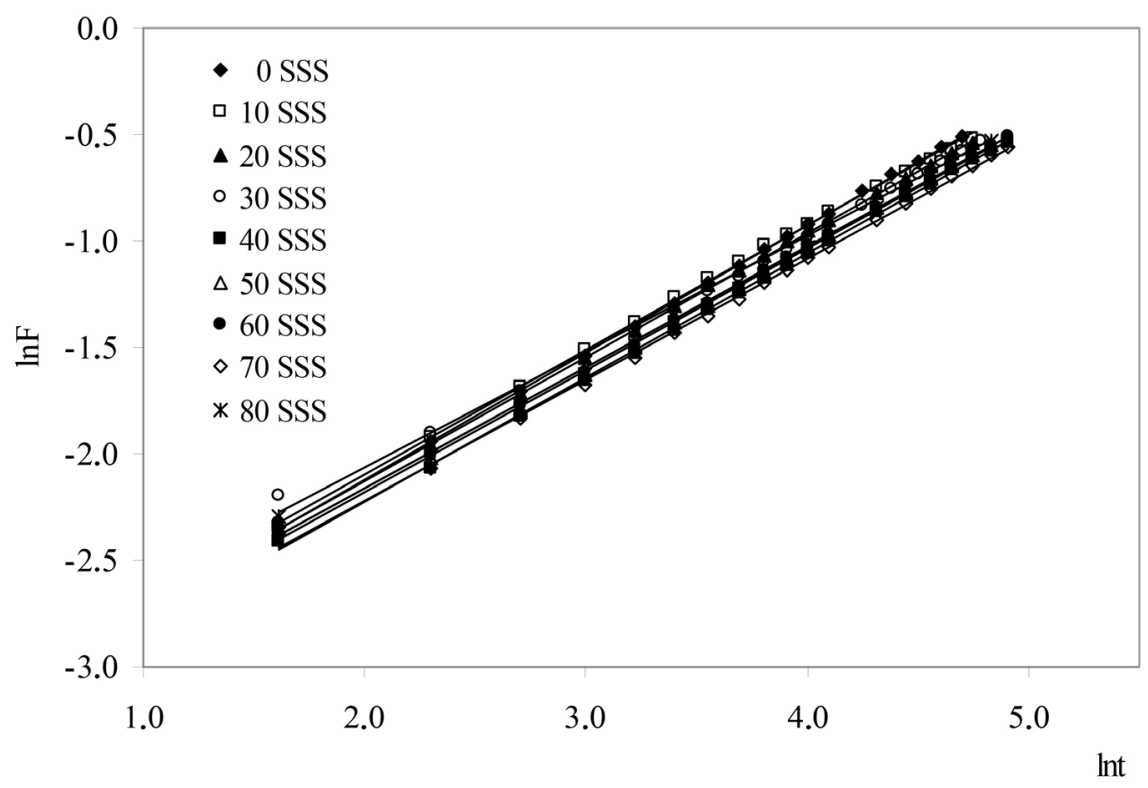

Figure 10. Swelling kinetic curves of AAm/SSS hydrogels. 


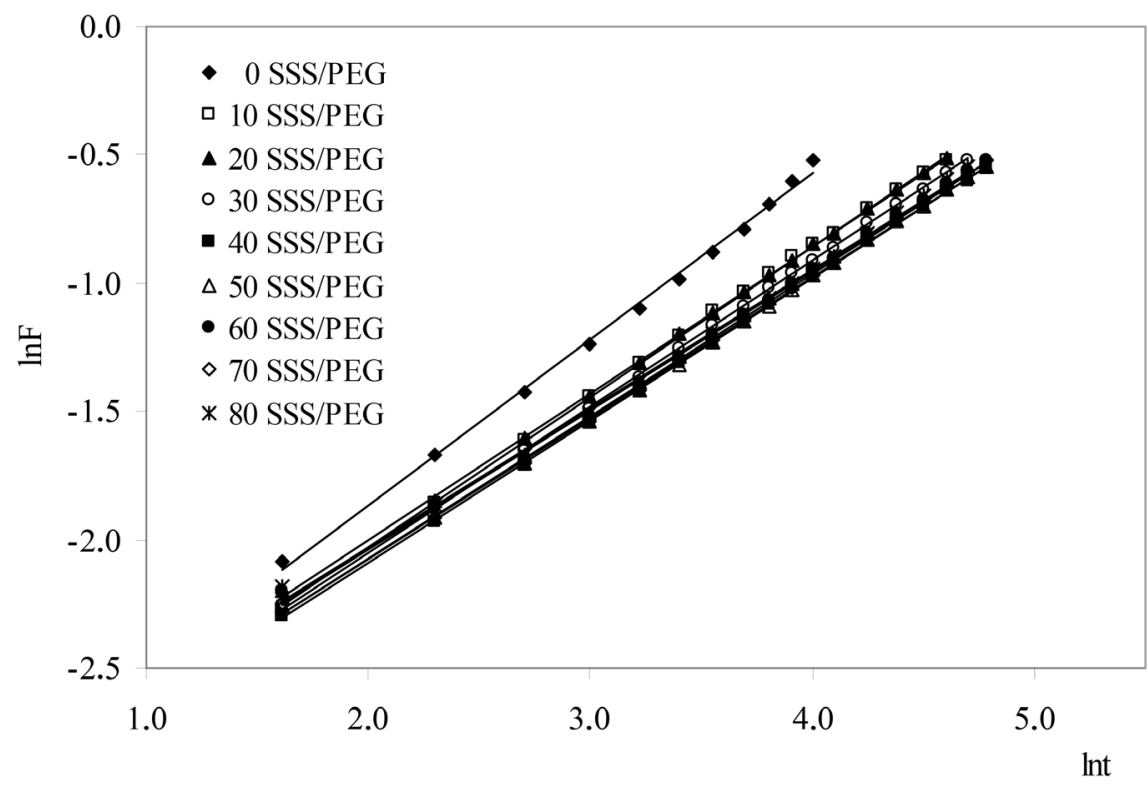

Figure 11. Swelling kinetic curves of AAm/SSS/PEG semi IPNs.

Table 4. Some diffusion parameters of AAm/SSS hydrogels and AAm/SSS/PEG (containing $0.5 \mathrm{~g}$ PEG) semi IPNs.

\begin{tabular}{|c|c|c|c|c|c|c|c|c|c|}
\hline $\mathrm{SSS} / \mathrm{mg}$ & 0 & 10 & 20 & 30 & 40 & 50 & 60 & 70 & 80 \\
\hline & \multicolumn{9}{|c|}{ Diffusion exponent $(n)$} \\
\hline & 0.5985 & 0.5841 & 0.5826 & 0.5425 & 0.5831 & 0.5749 & 0.5662 & 0.5685 & 0.5684 \\
\hline \multirow[t]{3}{*}{ PEG } & 0.6468 & 0.5781 & 0.5714 & 0.5672 & 0.5546 & 0.5498 & 0.5407 & 0.5550 & 0.5399 \\
\hline & \multicolumn{9}{|c|}{ Diffusion constant $\left(k \times 10^{2}\right)$} \\
\hline & 3.61 & 3.82 & 3.72 & 4.30 & 3.36 & 3.58 & 3.72 & 3.49 & 3.69 \\
\hline \multirow[t]{3}{*}{ PEG } & 4.24 & 3.55 & 4.31 & 4.15 & 4.08 & 4.17 & 4.44 & 4.14 & 4.48 \\
\hline & \multicolumn{9}{|c|}{ Diffusion coefficient $\left(D \times 10^{6}\right)$} \\
\hline & 159 & 114 & 129 & 96 & 109 & 125 & 115 & 110 & 144 \\
\hline PEG & 294 & 65 & 115 & 102 & 82 & 87 & 91 & 104 & 97 \\
\hline
\end{tabular}

0.50 . Hence the diffusion of water into the super water-retainer hydrogels is generally found to have a non-Fickian character. When the diffusion type is anomalous behavior, the relaxation and diffusion time are of the same order of magnitude. As solvent diffuses into the hydrogel, rearrangement of chains does not occur immediately.

The study of diffusion phenomena of water in hydrogels is of value in that it clarifies polymer behavior. For hydrogel characterization, the diffusion coefficients can be calculated by various methods. The diffusion coefficient, $(D)$ of the water was calculated using the following equation [30] [31].

$$
D=\pi r^{2}\left(\frac{k}{4}\right)^{1 / n}
$$

Here, $D$ is in $\mathrm{cm}^{2} \cdot \mathrm{min}^{-1}, r$ is the radius of a cylindrical polymer sample, $(n)$ is the 
diffusional exponent and $(k)$ is a constant incorporating characteristic of the macromolecular network system and the penetrant. The values of diffusion coefficient determined for AAm/SSS hydrogels and AAm/SSS/PEG semi IPNs are listed in Table 4. Table 4 shows that the values of the diffusion coefficient of AAm/SSS hydrogels and AAm/SSS/PEG semi IPNs vary from $65.0 \times 10^{-6}$ $\mathrm{cm}^{2} \cdot \mathrm{min}^{-1}$ to $294.0 \times 10^{-6} \mathrm{~cm}^{2} \cdot \mathrm{min}^{-1}$. There is no good correlation between the values of the diffusion coefficient of AAm/SSS hydrogels and AAm/SSS/PEG semi IPNs.

\subsection{PEG Effect on the Swelling and Diffusion}

For investigation of the effect of mass/content of PEG on the swelling properties of AAm/SSS/PEG semi IPNs, the related swelling isotherms of AAm/SSS/PEG hydrogels were constructed and representative swelling isotherms, swelling rate curves and the plot of $\ln F$ vs. $\ln t$ are plotted and representative results are shown in Figures 12-14. PEG effect on some swelling and diffusion parameters of AAm/SSS/PEG semi IPN systems containing $60 \mathrm{mg}$ SSS are tabulated in Table 5.

It was shown that a decreasing of the equilibrium percentage swelling, equilibrium water contents, diffusion constant, diffusion exponent, diffusion coefficient, initial swelling rate, swelling rate constant and theoretical equilibrium percentage swelling values of AAm/SSS/PEG semi IPN systems when PEG has been added to the hydrogel systems. Incorporation of PEG into the copolymer network leads to lower degrees of swelling. On the other hand, also, it is seen that an increasing diffusion constant $(k)$ of AAm/SSS/PEG hydrogels with increasing content of PEG in hydrogel systems from Table 5. Here, it was said that PEG chains were placed in the crosslinked polymeric systems, instead of crosslinked AAm and SSS molecules, it was seen that decreasing of the value of the

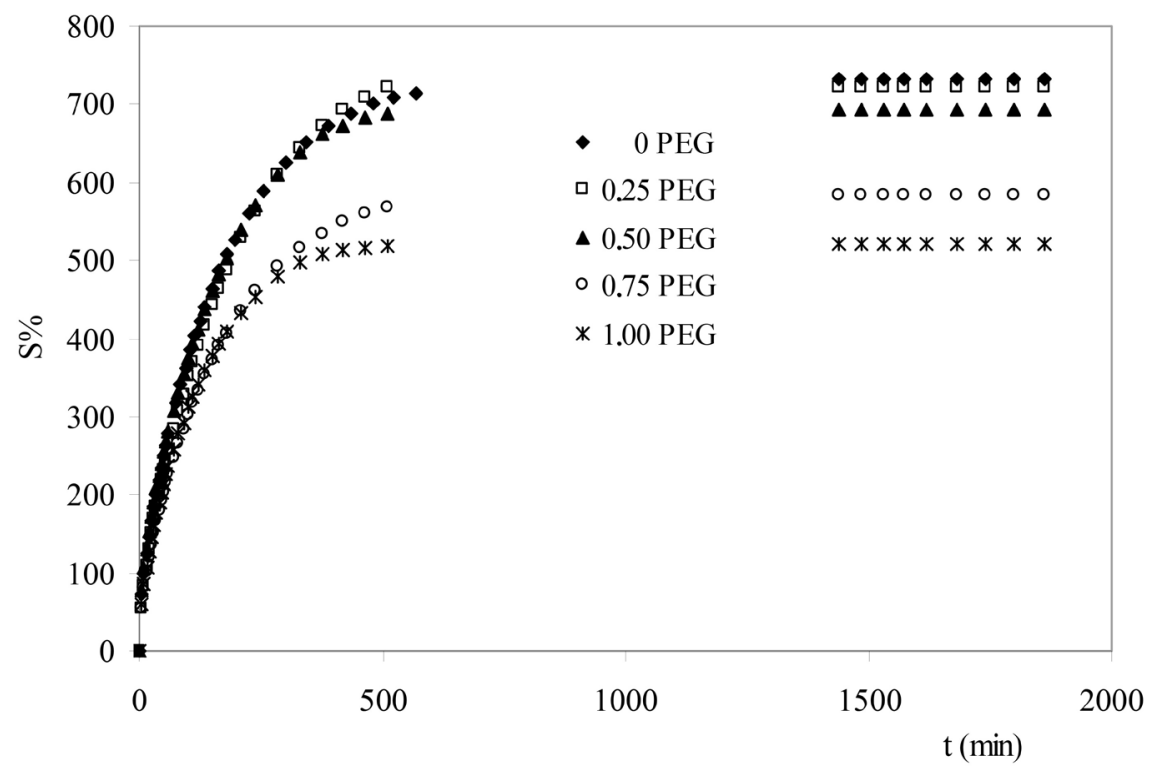

Figure 12. Swelling isotherms of AAm/SSS/PEG semi IPNs with various contents of PEG and containing $60 \mathrm{mg}$ SSS. 


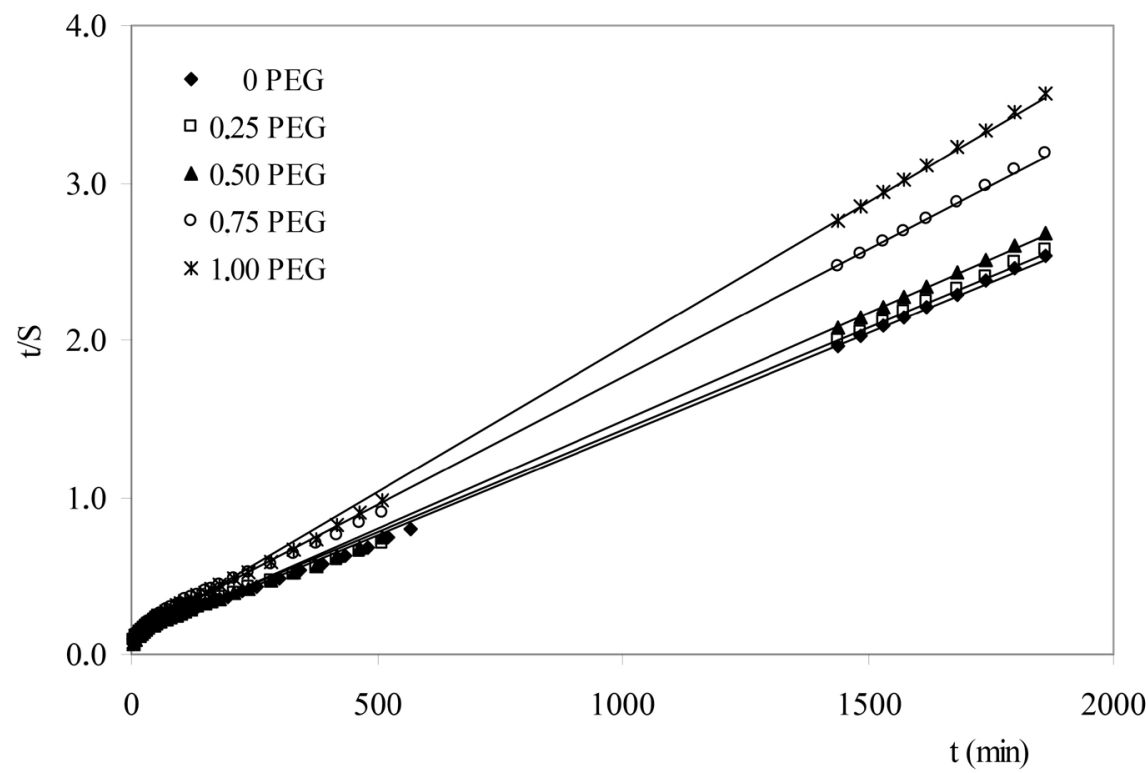

Figure 13. Swelling rate curves of AAm/SSS/PEG semi IPNs with various contents of PEG and containing $60 \mathrm{mg}$ SSS.

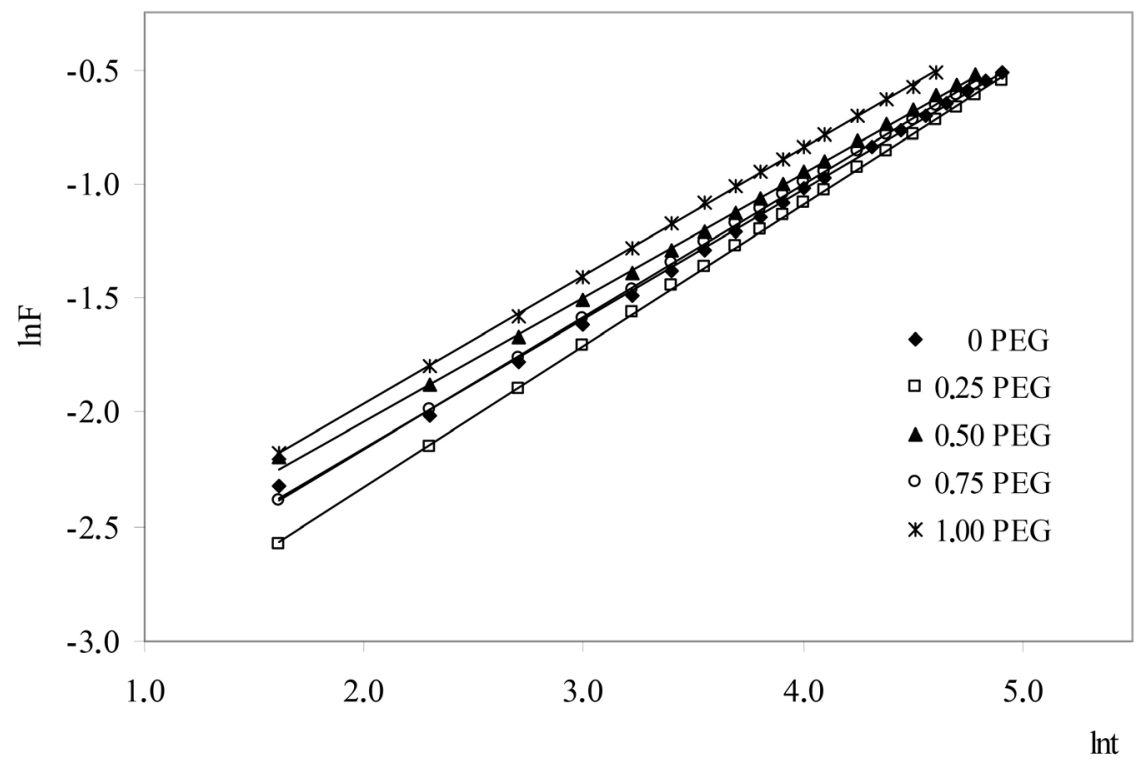

Figure 14. Swelling kinetic curves of AAm/SSS/PEG semi IPNs with various contents of PEG and containing $60 \mathrm{mg}$ SSS.

Table 5. Some swelling and diffusion parameters of AAm/SSS/PEG semi IPNs with various contents as gram of PEG and containing $60 \mathrm{mg}$ SSS.

\begin{tabular}{ccccc}
\hline 0 PEG & 0.25 PEG & 0.50 PEG & 0.75 PEG & 1.00 PEG \\
\hline \multirow{5}{*}{730} & \multicolumn{2}{c}{ Equilibrium } & percentage swelling $\left(S_{\text {eq }} \%\right)$ \\
& 720 & 690 & 580 & 520 \\
0.8800 & \multicolumn{2}{c}{ Equilibrium water content $($ EWC $)$} \\
\hline
\end{tabular}




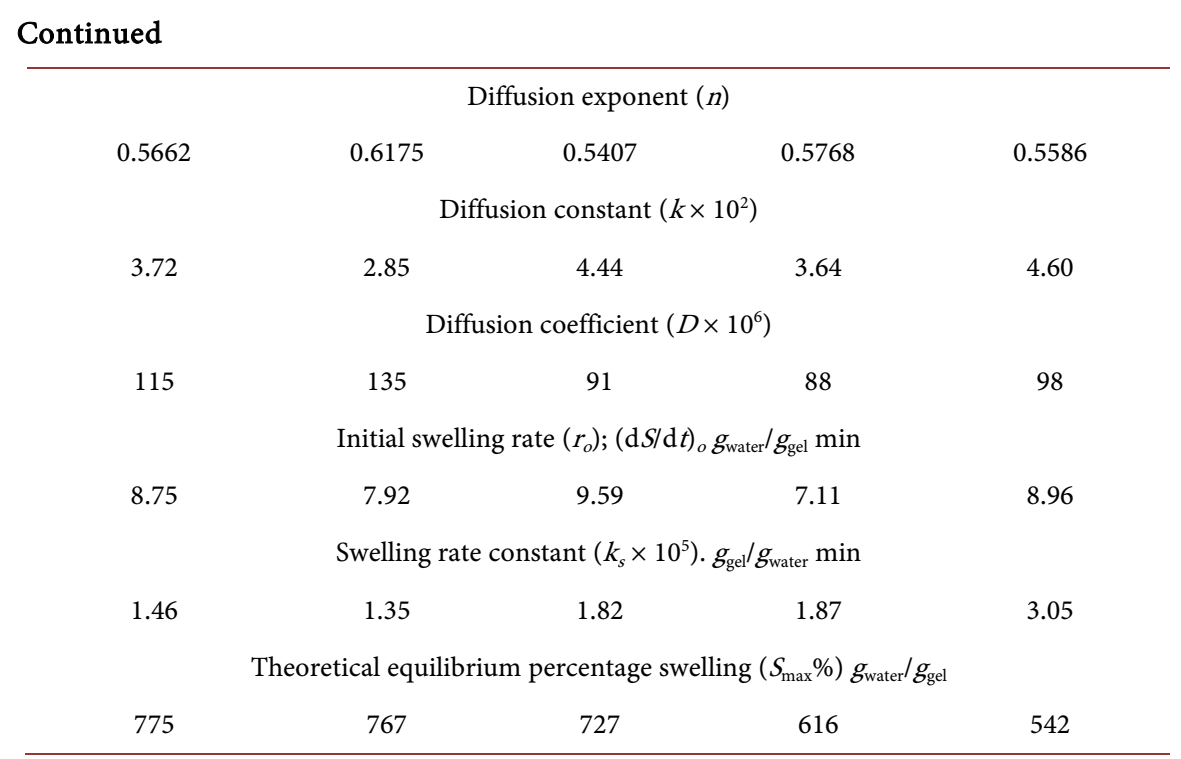

equilibrium swelling percent and related parameters, because of decreasing of hydrophilic character at crosslinked polymeric systems. In addition of this phenomenon, the PEG chains are located in the free space of crosslinked polymer networks; water diffusion is prevented by the PEG chains. This is also caused of decreasing of the equilibrium percentage swelling and related parameters.

\subsection{Scanning Electron Microscopy (SEM) Analysis}

SEM is the most used technique to investigate the shape, size, morphology, crosslink and porosity of hydrogels, or other related materials. Two SEM micrographs of the crosslinked copolymeric samples by PEGDA are presented in Figure 15 and Figure 16. If SSS is added to the hydrogel system, the observed crosslinked densities are very high (Figure 16). The micro-structural differentiations can be followed from Figure 15 and Figure 16. If Figure 16 is examined, it was seen that a lot of number of porous and crosslinked structure.

\subsection{Equilibrium Sorption Studies}

To observe the sorption of Janus Green B (JGB), AAm/SSS hydrogels and AAm/ SSS/PEG semi IPNs were placed in aqueous solutions of JGB and allowed to equilibrate for four days at $25^{\circ} \mathrm{C}$. At the end of this period AAm/SSS hydrogels and AAm/SSS/PEG semi IPNs in the JGB solutions showed the dark coloration. But acrylamide hydrogel did not sorb any dye from solution.

For equilibrium sorption studies, the dye removal capacity $(q)$ (mass amount as "mol" of sorption per unit mass (as gram) of the adsorbent, adsorption percentage (Ads\%), and partition coefficient $\left(K_{d}\right)$ can be investigated.

The dye removal capacity $(q)$ of AAm/SSS hydrogels and AAm/SSS/PEG semi IPNs were evaluated by using the following equation:

$$
q=\frac{\left(C_{o}-C\right) v}{m}
$$




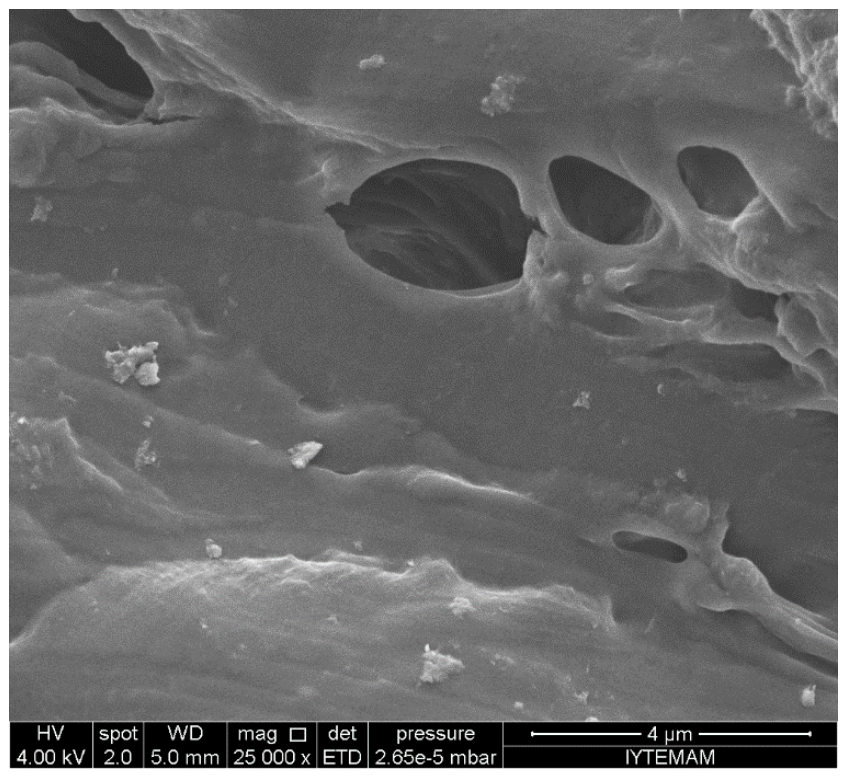

Figure 15. Scanning electron micrographs of AAm hydrogels.

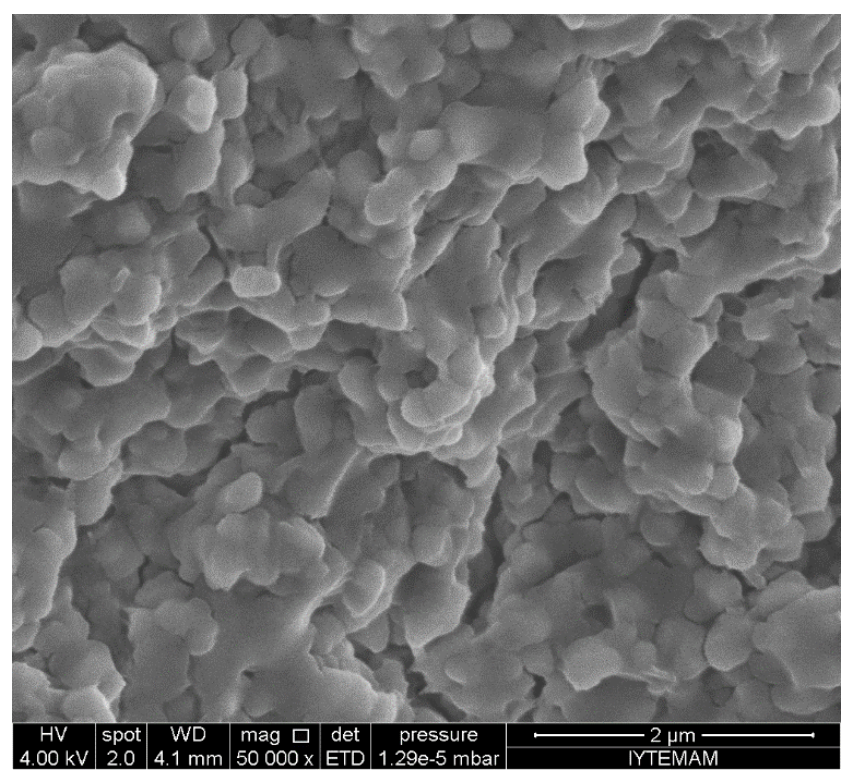

Figure 16. Scanning electron micrographs of AAm/SSS hydrogels containing of $60 \mathrm{mg}$ SSS.

where $q$ is the dye removal capacity of AAm/SSS hydrogels and AAm/SSS/PEG semi IPNs $\left(\mathrm{mol} \cdot \mathrm{g}^{-1}\right), C_{o}$ and $C$ are the concentration of the dye in the initial solution and the aqueous phase after treatment for a certain period time, respectively $\left(\mathrm{mol} \cdot \mathrm{L}^{-1}\right), v$ is the volume of the aqueous phase $(L), \mathrm{m}$ is the amount of dry AAm/SSS hydrogels and AAm/SSS/PEG semi IPNs.

Adsorption percentage (Ads\%) of AAm/SSS hydrogels and AAm/SSS/PEG semi IPNs was calculated by following equation.

$$
\operatorname{Ads} \%=\frac{C_{o}-C}{C_{o}} \times 100
$$


Here $C_{o}$ and $C$ were defined earlier.

The effect of SSS contents onto dye uptake capacity was measured. The dye removal capacity, the amount of dyes sorbed onto unit dry mass of the gel was calculated for uptake of dye within the hydrogel in $2.00 \times 10^{-3}$ mol JGB in L of aqueous solutions, and presented in Table 6 . Table 6 presents that the dye removal capacity of AAm/SSS hydrogels and AAm/SSS/PEG semi IPNs $(1.35 \times$ $\left.10^{-4}-4.12 \times 10^{-4} \mathrm{~mol} \cdot \mathrm{g}^{-1}\right)$ and adsorption percentage of these $(20 \%-74 \%)$ both are increased with together. The dye removal capacity and adsorption percentage of AAm/SSS hydrogels and AAm/SSS/PEG semi IPNs gradually increased with increasing of SSS content in hydrogels and semi IPNs.

Equilibrium JGB sorption isotherms of AAm/SSS hydrogels and AAm/SSS/PEG semi IPNs are presented in Figure 17 \& Figure 18. To Figure 17 \& Figure 18, the sorption capacity of the hydrogel systems is increased with the increasing concentration of JGB. This is expected result.

Partitioning of dissolved constituents between an aqueous phase and adsorbents in waters and sediments has commonly been described by an empirical partition coefficient that simply relates the total concentration of a dissolved species to the total concentration of the adsorbed species. For this parameter, the given equation at below can be used [32] [33].

$$
K_{d}=\frac{C_{o}-C}{C}
$$

Here; $K_{d}$ is empirical partition coefficient at equilibrium. $C_{o}$ and $C$ were defined earlier. Partition coefficients of JGB between dye solution and hydrogels were calculated, and are shown in Table 6. In Table 6, $K_{d}$ values of AAm/SSS hydrogels is $0.32-2.85$, but $K_{d}$ values of AAm/SSS/PEG semi IPNs is $0.25-1.54$ with the incorporation of PEG groups into the hydrogels.

Here, $K_{d}$ values of AAm/SSS hydrogels and AAm/SSS/PEG semi IPNs (having $60 \mathrm{mg}$ and more than this SSS content) are higher than 1.0. So, it can be said that synthesized crosslinked AAm/SSS hydrogels and AAm/SSS/PEG semi IPNs (having

Table 6. Some adsorption parameters of AAm/SSS hydrogels and AAm/SSS/PEG (containing $60 \mathrm{mg}$ SSS and $0.5 \mathrm{~g}$ PEG) semi-IPNs in aqueous solutions of JGB.

\begin{tabular}{|c|c|c|c|c|c|c|c|c|}
\hline $\mathrm{SSS} / \mathrm{mg}$ & 10 & 20 & 30 & 40 & 50 & 60 & 70 & 80 \\
\hline & \multicolumn{8}{|c|}{ Dye removal capacity $\left(q \times 10^{4}\right)$} \\
\hline & 1.35 & 1.88 & 2.09 & 2.76 & 2.93 & 3.47 & 3.72 & 4.12 \\
\hline \multirow[t]{3}{*}{ PEG } & 1.43 & 1.56 & 2.39 & 2.66 & 3.09 & 3.44 & 3.51 & 3.97 \\
\hline & \multicolumn{8}{|c|}{ Adsorption percentage (Ads\%) } \\
\hline & 24 & 32 & 35 & 45 & 46 & 55 & 54 & 74 \\
\hline \multirow[t]{3}{*}{ PEG } & 20 & 24 & 36 & 38 & 47 & 51 & 58 & 61 \\
\hline & \multicolumn{8}{|c|}{ Partition coefficient $\left(K_{d}\right)$} \\
\hline & 0.32 & 0.46 & 0.53 & 0.81 & 0.86 & 1.21 & 1.17 & 2.85 \\
\hline PEG & 0.25 & 0.32 & 0.57 & 0.63 & 0.87 & 1.05 & 1.41 & 1.54 \\
\hline
\end{tabular}




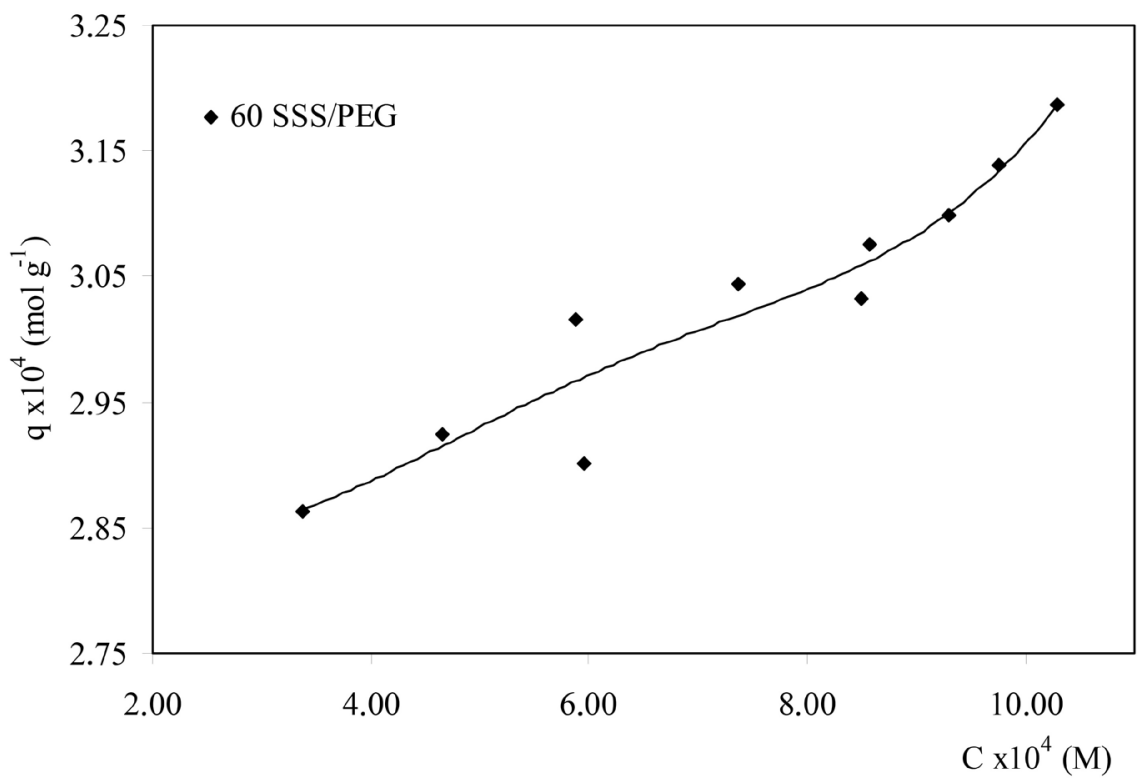

Figure 17. Equilibrium sorption isotherms of AAm/SSS hydrogels in aqueous JGB solutions.

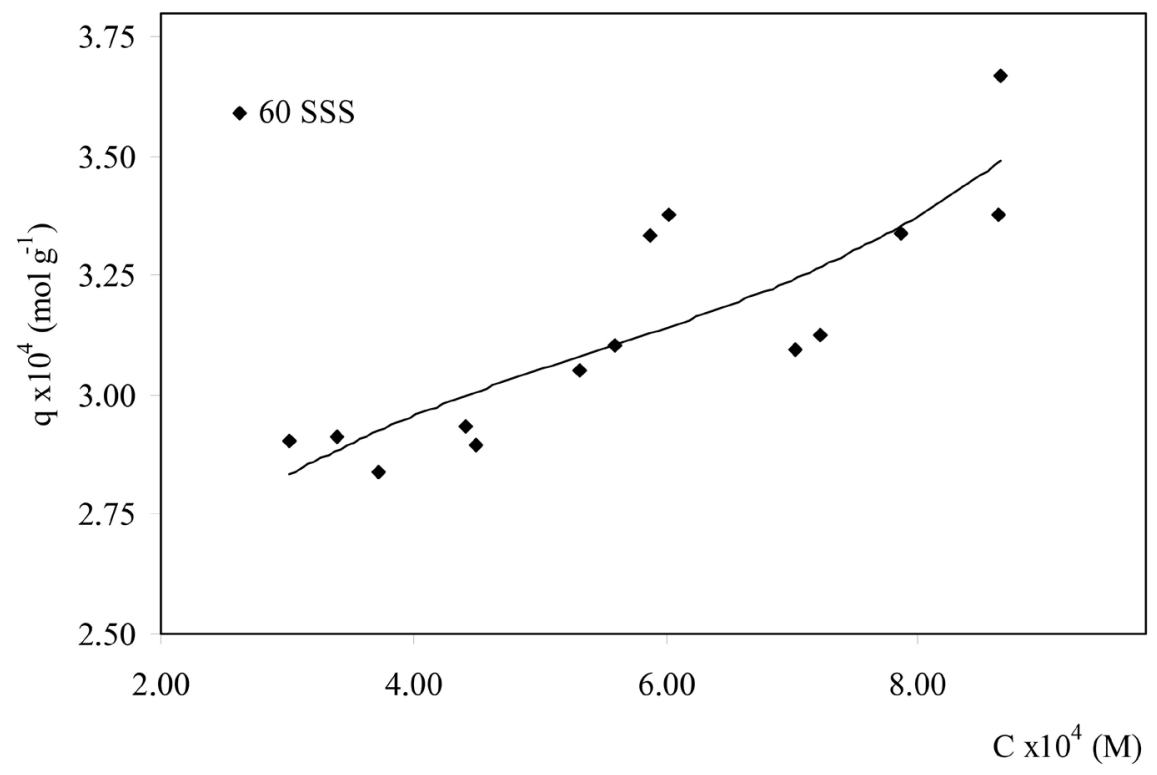

Figure 18. Equilibrium sorption isotherms of AAm/SSS/PEG semi IPNs in aqueous JGB solutions.

$60 \mathrm{mg}$ and more than this SSS content) could be used as potential adsorbent [32] [33].

\subsection{PEG Effect on the Sorption of JGB}

For investigation of the effect of mass/content of PEG on the sorption properties of AAm/SSS/PEG semi IPNs, some sorption parameters such as dye removal capacity, adsorption percentage and partition coefficient of AAm/SSS/PEG semi IPN systems containing $60 \mathrm{mg}$ SSS are tabulated in Table 7. 
Table 7. PEG effect on some adsorption parameters of AAm/SSS/PEG semi IPNs with various contents as gram of PEG and containing $60 \mathrm{mg}$ SSS in aqueous solutions of JGB.

\begin{tabular}{|c|c|c|c|c|c|}
\hline $60 \mathrm{mg}$ SSS & 0 PEG & 0.25 PEG & $0.50 \mathrm{PEG}$ & 0.75 PEG & 1.00 PEG \\
\hline & \multicolumn{5}{|c|}{ Dye removal capacity $\left(q \times 10^{4}\right)$} \\
\hline & 3.47 & 3.29 & 3.44 & 3.16 & 3.43 \\
\hline & \multicolumn{5}{|c|}{ Adsorption percentage (Ads\%) } \\
\hline & 55 & 54 & 51 & 50 & 44 \\
\hline & \multicolumn{5}{|c|}{ Partition coefficient $\left(K_{d}\right)$} \\
\hline & 1.21 & 1.16 & 1.05 & 1.01 & 0.78 \\
\hline
\end{tabular}

It was shown that a decreasing of adsorption percentage (55\% - 44\%) and partition coefficient (1.21 - 0.78) of AAm/SSS/PEG semi IPN systems when PEG has been added to the hydrogel systems. Incorporation of PEG into the copolymer network leads to lower values of adsorption percentage and partition coefficient of AAm/SSS/PEG semi IPN systems. Here, $K_{d}$ values of AAm/SSS/PEG semi IPNs including $0 \mathrm{~g}, 0.25 \mathrm{~g}, 0.5 \mathrm{~g}$ and $0.75 \mathrm{~g}$ of PEG are higher than 1.0 (1.21 - 1.01). So, it can be said that synthesized crosslinked AAm/SSS/PEG semi IPNs (having $60 \mathrm{mg}$ SSS and $0.0 \mathrm{~g}-0.75 \mathrm{~g}$ PEG content) could be used as potential adsorbent. On the other hand, it can be said that there is no important changing of the dye removal capacity of AAm/SSS/PEG semi IPN systems when PEG has been added to the hydrogel systems $\left(3.16 \times 10^{-4}-3.47 \times 10^{-4} \mathrm{~mol} \cdot \mathrm{g}^{-1}\right)$.

Here, it was said that PEG chains were placed in the crosslinked polymeric systems, instead of crosslinked AAm and SSS molecules, it was seen that decreasing of the adsorption percentage, because of decreasing of hydrophilic character at crosslinked polymeric systems. In addition of this phenomenon, the PEG chains are located in the free space of crosslinked polymer networks; JGB sorption is prevented by the PEG chains. This is also caused by partition decreasing of the adsorption percentage.

The ionic charge content in the polymeric structure is important. SSS contains ionic units $\left(-\mathrm{SO}_{3} \mathrm{Na}\right)$. The swelling degrees of the hydrogels increase due to increasing of the hydrophilic units on hydrogel structure (Figure 1). Therefore, AAm/SSS hydrogels and AAm/SSS/PEG semi IPNs have many ionic groups that can increase interaction between the cationic dye molecules and anionic groups of hydrogels. The results of swelling studies are parallel character to the results of sorption studies. Both of them, it can be seen that swelling or sorption capability of AAm/SSS hydrogels and AAm/SSS/PEG semi IPNs are increased with increasing SSS content in copolymeric structure. The most important effect is hydrophilicity of copolymeric gels. Hydrophilicity of AAm/SSS hydrogels and AAm/SSS/PEG semi IPNs becomes greater than that of AAm hydrogels, when addition of SSS to the crosslinked copolymeric structure.

For good binding analysis, Table 8 can be arranged. There can be many reasons for non-covalent interactions in the binding of JGB by AAm/SSS hydrogels and AAm/SSS/PEG semi IPNs. The main interactions between the hydrogel and 
Table 8. Possible non-covalent interactions in the binding of JGB by AAm/SSS hydrogels.

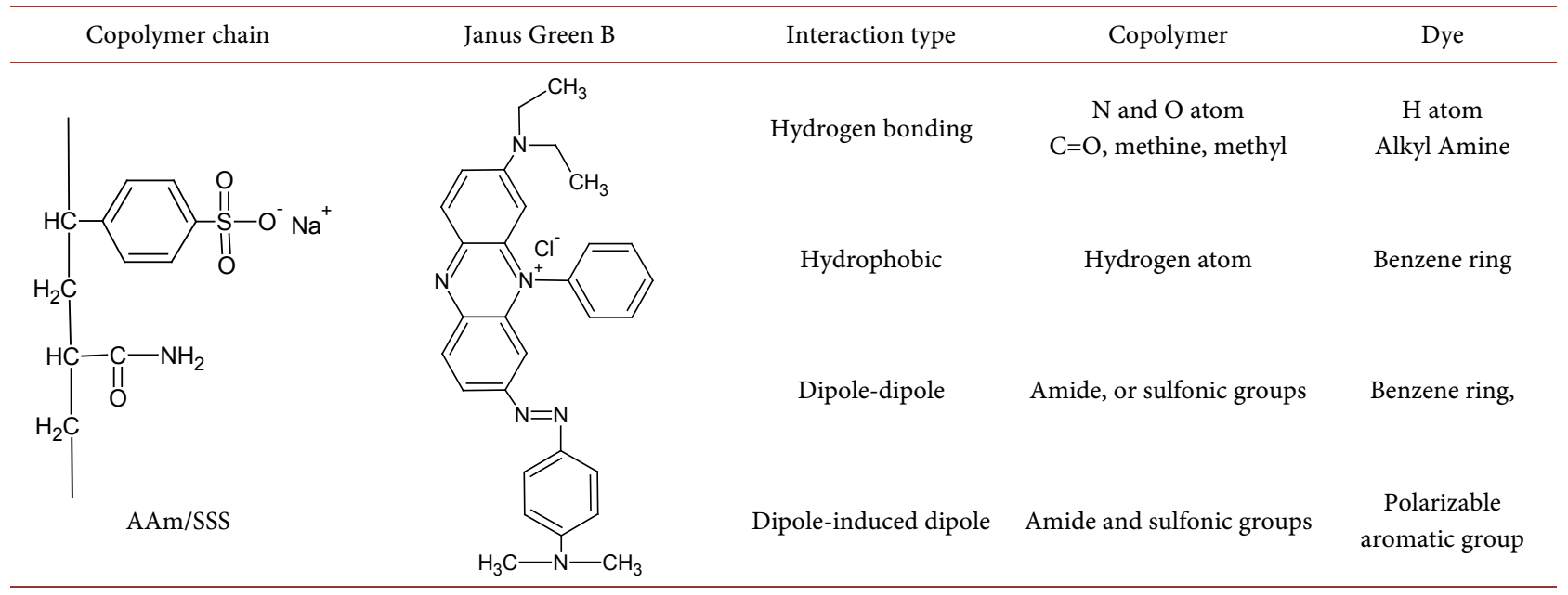

the monovalent cationic dye may be hydrophobic and hydrogen bonding. Specially, hydrogen bonding will be expected to occur between amine groups and nitrogen atoms on the dye molecules and the amine and carbonyl groups on the monomer unit of crosslinked polymer. Hydrophobic effects are especially aqueous solutions interactions which in the present case will involve those aromatic rings on the dye molecules and the methine and methyl groups on the gel. There can be some other interactions such as dipole-dipole and dipole-induced dipole interactions between the dye molecules and the hydrogel chains.

\section{Conclusions}

Incorporation of the hydrophilic group containing chemicals such as SSS and a polymer such as PEG in AAm hydrogels can be obtained successively by the free radical solution polymerization method. Multifunctional crosslinker such as PEGDA is used in the polymerization process. AAm/SSS hydrogels and AAm/ SSS/PEG semi IPNs showed high water absorbency. The equilibrium percentage swelling ranges are $490 \%-850 \%$ for AAm/SSS hydrogels and $420 \%-790 \%$ for AAm/SSS/PEG semi IPNs. It was seen that swelling of AAm/SSS hydrogels and AAm/SSS/PEG semi IPNs increased with the increasing of content of SSS. But, it was seen that a decreasing of values of $S_{e q} \%$ from $730 \%$ to $520 \%$ when the adding of PEG for containing $60 \mathrm{mg}$ of SSS.

To determine the sorption characteristics of JGB into AAm/SSS hydrogels and AAm/SSS/PEG semi IPNs, some sorption parameters have been investigated. For equilibrium sorption studies, dye removal capacity, adsorption percentage, and partition coefficient of AAm/SSS hydrogels and AAm/SSS/PEG semi IPNs system have been investigated. Dye removal capacity values of the hydrogel systems were changed between $1.35 \times 10^{-4}-4.12 \times 10^{-4} \mathrm{~mol} \cdot \mathrm{g}^{-1}$. The values of Ads $\%$ of the hydrogels were changed among $20 \%-74 \%$. The values of $K_{d}$ of the hydrogels $0.25-2.85$.

Consequently, AAm/SSS hydrogels and AAm/SSS/PEG semi IPNs developed 
in this study may serve as a potential device for water and dye sorbent. The utilization of these types of materials, in pharmaceuticals, agriculture, biotechnology, environment, separation, purification, and immobilization makes hydrogels more popular.

\section{Acknowledgements}

The work was supported by Adnan Menderes University Research Fund, under project number FEF 08034 .

\section{Conflicts of Interest}

No potential conflict of interest was reported by the authors.

\section{References}

[1] Hassan, T., Salam, A., Khan, A., Khan, S.U., Khanzada, H., Wasim, M., Khan, M.O. and Kim, I.S. (2021) Functional Nanocomposites and Their Potential Applications: A Review. Journal of Polymer Research, 28, 36. https://doi.org/10.1007/s10965-021-02408-1

[2] Jaspal, D. and Malviya, A. (2020) Composites for Wastewater Purification: A Review. Chemosphere, 246, Article ID: 125788. https://doi.org/10.1016/j.chemosphere.2019.125788

[3] Karadağ, E., Öztürk, Z.D.K., Üzüm, Ö.B. and Kundakc1, S. (2019) Swelling Performance Studies of Acrylamide/Potassium 3-Sulfopropyl Methacrylate/Sodium Alginate/Bentonite Biohybrid Sorbent Hydrogels in Binary Mixtures of Water-Solvent. Journal of Encapsulation and Adsorption Sciences, 9, 35-61. https://doi.org/10.4236/jeas.2019.91003

[4] Akharame, M.O., Fatoki, O.S., Opeolu, B.O., Olorunfemi, D.I. and Oputu, O.U. (2018) Polymeric Nanocomposites (PNCs) for Wastewater Remediation: An Overview. Polymer-Plastics Technology and Engineering, 57, 1801-1827. https://doi.org/10.1080/03602559.2018.1434666

[5] Urbano, B. and Rivas, B.L. (2011) Poly(sodium 4-styrene sulfonate) and Poly(2acrylamidoglycolic acid) Nanocomposite Hydrogels: Montmorillonite Effect on Water Absorption, Thermal, and Rheological Properties. Polymer Bulletin, 67, 1823 1836. https://doi.org/10.1007/s00289-011-0511-2

[6] Kundakcı, S., Öğüt, H.G., Üzüm, Ö.B. and Karadağ, E. (2011) Equilibrium Swelling Characterization and Dye Uptake Studies of Acrylamide-co-methylenesuccinic Acid Hydrogels and Semi-IPN's with PEG. Polymer-Plastics Technology and Engineering, 50, 947-956. https://doi.org/10.1080/03602559.2011.553862

[7] Mohan, Y.M., Sudhakar, K., Murty, K.P.S. and Raju, K.M. (2006) Swelling Properties of Chemically Crosslinked Poly(acrylamide-co-maleic acid) Hydrogels. International Journal of Polymeric Materials, 55, 513-536. https://doi.org/10.1080/00914030500208246

[8] Şahiner, N., Godbey, W.T., McPerson, G.L. and John, V.T. (2006) Microgel, Nanogel and Hydrogel-Hydrogel Semi-IPN Composites for Biomedical Applications: Synthesis and Characterization. Colloid and Polymer Science, 284, 1121-1129. https://doi.org/10.1007/s00396-006-1489-4

[9] Kosmala, J.D., Henthorn, D.B. and Brannon-Peppas, L. (2000) Preparation of Interpenetrating Network of Gelatin and Dextran as Degradable Biomaterials. Bioma- 
terials, 21, 2019-2023. https://doi.org/10.1016/S0142-9612(00)00057-0

[10] Şahiner, N. (2007) Colloidal Nanocomposite Hydrogel Particles. Colloid and Polymer Science, 285, 413-421. https://doi.org/10.1007/s00396-006-1583-7

[11] Sperling, L.H. (1994) Interpenetrating Polymer Networks: An Overwiew. Advances in Chemistry, American Chemical Society, Washington DC, 1-37. https://doi.org/10.1021/ba-1994-0239.ch001

[12] Üzüm, Ö.B. and Karadağ, E. (2010) Equilibrium Swelling Studies of Chemically Crosslinked Highly Swollen Acrylamide-Sodium Acrylate Hydrogels in Various Water-Solvent Mixtures. Polymer-Plastics Technology and Engineering, 49, 609616. https://doi.org/10.1080/03602551003664537

[13] Üzüm, Ö.B., Kundakc1, S. and Karadağ, E. (2009) Equilibrium Swelling Studies of Highly Swollen Acrylamide/Thiosinamine Hydrogels. Polymer-Plastics Technology and Engineering, 48, 152-157. https://doi.org/10.1080/03602550802577338

[14] Wang, Y., Zeng, Li., Ren, X., Song, H. and Wang, A. (2010) Removal of Methyl Violet from Aqueous Solutions Using Poly(acrylic acid-co-acrylamide)/Attapulgite Composite. Journal of Environmental Sciences, 22, 7-14. https://doi.org/10.1016/S1001-0742(09)60068-1

[15] Üzüm, Ö.B. and Karadağ, E. (2006) Synthetic Polymeric Absorbent for Dye Based on Chemically Crosslinked Acrylamide/Mesaconic Acid Hydrogels. Journal of Applied Polymer Science, 101, 405-413. https://doi.org/10.1002/app.22248

[16] Li, W., Zhao, H., Teasdale, P.R., John, R. and Zhang, S. (2002) Synthesis and Characterisation of a Polyacrylamide-Polyacrylic Acid Copolymer Hydrogel for Environmental Analysis of $\mathrm{Cu}$ and Cd. Reactive \& Functional Polymers, 52, 31-41. https://doi.org/10.1016/S1381-5148(02)00055-X

[17] İnam, R., Çaykara, T. and Kantoğlu, Ö. (2003) Polarographic Determination of Uranyl Adsorption onto Poly(acrylamide-g-ethylenediaminetetraacetic acid) Hydrogels in the Presence of Cadmium and Lead. Nuclear Instruments and Methods in Physics Research Section B, 208, 400-404. https://doi.org/10.1016/S0168-583X(03)01110-8

[18] Rivas, B.L. and Munoz, C. (2009) Synthesis and Metal Ion Adsorption Properties of Poly(4-sodium styrene sulfonate-co-acrylic acid). Journal of Applied Polymer Science, 114, 1587-1592. https://doi.org/10.1002/app.30722

[19] Karadağ, E., Kundakc1, S. and Üzüm, Ö.B. (2009) Water Sorption and Dye Uptake Studies of Highly Swollen AAm/AMPS Hydrogels and Semi-IPN's with PEG. PolymerPlastics Technology and Engineering, 48, 1217-1229. https://doi.org/10.1080/03602550903159044

[20] Krsko, P. and Libera, M. (2005) Biointeractive Hydrogels. Materials Today, 8, 36-44. https://doi.org/10.1016/S1369-7021(05)71223-2

[21] Serra, L., Domenech, J. and Peppas, N.A. (2006) Design of Poly(ethylene glycol)Tethered Copolymers as Novel Mucoadhesive Drug Delivery Systems. European Journal of Pharmaceutics and Biopharmaceutics, 63, 11-18. https://doi.org/10.1016/j.ejpb.2005.10.011

[22] Üzüm, Ö.B. and Karadağ, E. (2011) Dye Sorption and Water Uptake Properties of Crosslinked Acrylamide/Sodium Methacrylate Copolymers and Semi-Interpenetrating Polymer Networks Composed of PEG. Separation Science and Technology, 46, 489499. https://doi.org/10.1080/01496395.2010.513697

[23] Skoog, D.A. and Leary, J.J. (1992) Principles of Instrumental Analysis. Saunders College Publishing, Philadelphia, 279. 
[24] Lee, S.J., Kim, S.S. and Lee, Y.M. (2000) Interpenetrating Polymer Network Hydrogels Based an Poly(ethylene glycol) Macromer and Chitosan. Carbohydrate Polymers, 41, 197-205. https://doi.org/10.1016/S0144-8617(99)00088-0

[25] Kim, S.J., Park, S.J. and Kim, S.I. (2003) Synthesis and Characterization of Interpenetrating Polymer Network Hydrogels Composed of Poly(vinyl alcohol) and Poly(Nisopropylacrylamide). Reactive \& Functional Polymers, 55, 61-67. https://doi.org/10.1016/S1381-5148(02)00215-8

[26] Azizian, S. (2004) Kinetic Models of Sorption: A Theoretical Analysis. Journal of Colloid and Interface Science, 276, 47-52. https://doi.org/10.1016/j.jcis.2004.03.048

[27] Saraydin, D., Karadağ, E., Işıkver, Y., Şahiner, N. and Güven, O. (2004) The Influence of Preparation Methods on the Swelling and Network Properties of Acrylamide Hydrogels with Crosslinkers. Journal of Macromolecular Science, Part A: Pure and Applied Chemistry, 41, 421-433. https://doi.org/10.1081/MA-120028476

[28] Peppas, N.A. and Franson, N.M. (1983) The Swelling Interface Number as a Criterion for Prediction of Diffusional Solute Release Mechanisms in Swellable Polymers. Journal of Polymer Science, 21, 983-997. https://doi.org/10.1002/pol.1983.180210614

[29] Am Ende, M.T. and Peppas, N.A. (1997) Transport of Ionizable Drugs and Proteins in Crosslinked Poly(acrylic acid) and Poly(acrylic acid-co-2-hydroxyethyl methacrylate) Hydrogels. II. Diffusion and Release Studies. Journal of Controlled Release, 48, 47-56. https://doi.org/10.1016/S0168-3659(97)00032-1

[30] Dengre, R., Bajpai, M. and Bajpai, S.K. (2000) Release of Vitamin B12 from Poly(Nvinyl-2-pyrrolidione)-crosslinked Polyacrylamide Hydrogels. Journal of Applied Polymer Science, 76, 1706-1714.

https://doi.org/10.1002/(SICI)1097-4628(20000613)76:11<1706::AID-APP12>3.0.C $\mathrm{O} ; 2-\mathrm{W}$

[31] Çaykara, T., Kiper, S. and Demirel, G. (2006) Thermosensitive Poly(Nisopropilacrylamide-co-acrylamide) Hydrogels: Synthesis, Swelling and Interaction with Ionic Surfactants. European Polymer Journal, 42, 348-355. https://doi.org/10.1016/j.eurpolymj.2005.07.006

[32] Schwarte, L.M. and Peppas, N.A. (1998) Novel Poly(ethylene glycol)-Grafted Cationic Hydrogels: Preparation, Characterization and Diffusive Properties. Polymer, 39, 6057-6066. https://doi.org/10.1016/S0032-3861(98)00087-1

[33] Şahiner, N., Saraydın, D., Karadağ, E. and Güven, O. (1998) Swelling and Dye Adsorption Properties of Radiation Induced N-vinyl-2-pyrrolidone/Acrylonitrile Hydrogels. Polymer Bulletin, 41, 371-378. https://doi.org/10.1007/s002890050376 